\title{
Evaluación radiográfica de la presentación de displasia de cadera en canes de la raza Mastín Napolitano en Lima Metropolitana.
}

\author{
Radiographic evaluation of hip dysplasia in Mastin Napolitano breed dogs in Lima.
}

\author{
Eben Salinas ${ }^{1}$, Edith Chávez ${ }^{2}$
}

RESUMEN

La displasia de cadera es la principal enfermedad ortopédica del desarrollo de los caninos, y se presenta generalmente en perros de tamaño grande o gigante. Esta enfermedad de forma progresiva degenerará a una enfermedad articular degenerativa. El presente estudio, mediante el estudio radiográfico, determina y describe la presentación de displasia de cadera en canes de raza Mastín Napolitano. Para tal fin se utilizaron ejemplares mayores de 6 meses de edad, en los cuales adicionalmente se evaluó la asociación de la enfermedad con variables demográficas: sexo, edad y signos clínicos. Se utilizaron los protocolos radiográficos planteados por la Federación Cinológica Internacional. El presente estudio incluyó 60 animales distribuidos en 30 canes por cada sexo los cuales fueron organizados en tres estratos etáreos. Se obtuvo un 65\% (39/60), de frecuencia de presentación de displasia de cadera, la cual resultó elevada al compararla con los antecedentes de la raza. No se obtuvo asociación entre la presentación de la enfermedad y las variables sexo y edad animal. La enfermedad se presentó en mayor proporción en caninos de 6 a 18 meses de edad, fue mayormente bilateral $(69,2 \%)$ y altamente asociada a signos clínicos $(63 \%)$. Finalmente el grado de displasia D (Media) fue el más frecuente en los animales evaluados. Estos resultados confirman la necesidad del examen radiológico para el diagnóstico preciso de la enfermedad en una raza históricamente susceptible.

PALABRAS CLAVE: Ángulo de Norberg, displasia de cadera, radiología, molosoide.

\section{SUMMARY}

Hip dysplasia it is a main orthopedic disease in the dog development and it is shown in big size dogs. This disease progressively degenerate to a degenerative joint disease. This study focused on determining and describing the presentation of hip dysplasia by radiographic study, in dogs of Mastin Napolitane breed and on the establishment of associations between disease and the demographic variables: sex, age and clinical signs. For this purpose, specimens older than 6 months of age were used, in which radiographic protocols proposed by the International Cynological Federation were used. For this purpose 60 animals were separated by sex $(n=30)$ and organized into three age strata. The results showed $65 \%$ (39/60) of hip dysplasia presentation frequency, being higher than that described in previous antecedents of the same race. No association was found between the presentation of the disease and the variables sex and animal age. The disease occurred in a greater proportion in canines from 6 to 18 months of age, being mostly bilateral (69.2\%) and highly associated with clinical signs (63\%). Finally, the degree of dysplasia D (medium) was the most frequent in the animals evaluated. These results confirm the need for radiological examination for accurate diagnosis of the disease in a historically susceptible race.

KEY WORDS: Norberg angle, hip dysplasia, radiology, molossoid.

Carrera de Medicina Veterinaria y Zootecnia, Universidad Científica del Sur. Lima, Perú

Clínica Veterinaria Doctor Pet's. Lima, Perú 


\section{INTRODUCCIÓN}

La displasia de cadera (DC) es la principal enfermedad ortopédica que se relaciona con el crecimiento y desarrollo de los caninos, se caracteriza por laxitud y pérdida de conformación articular de la articulación coxofemoral. Con la evolución del cuadro, se desarrollará como consecuencia, una enfermedad articular degenerativa (Ginja, Silvestre, GonzaloOrden y Ferreira, 2010). Esta es una enfermedad común en muchas razas de perros, especialmente grandes y gigantes. La DC es la principal causa de retiro de perros militares y la causa del 5\% de las eutanasias en perros en países europeos (Woolliams, Lewis y Blott, 2011).

La DC tiene una etiología multifactorial con factores desencadenantes como la nutrición, edad, sexo, el peso corporal, factores ambientales y ejercicio. Estas condiciones juegan un rol importante en la presentación de la enfermedad (Malm et al., 2010; Witsberger, Villamil, Schultz, Hahn y Cook, 2008). También se describe la excesiva laxitud de la articulación de la cadera por una baja calidad del tejido conectivo de la articulación de la capsula como la más común (Flückiger, 2007; Smith, 1997). Además se considera que las anormalidades de la articulación de la cadera son secundarias a otras anomalías físicas o a una deficiente masa muscular pélvica, ya que la articulación coxofemoral depende de la musculatura para mantener las superficies articulares durante el desarrollo (Cardinet, Kass, Wallace y Guffy, 1997).

De manera especial el tamaño y peso tienen una alta influencia sobre el desarrollo de la DC. En relación a este hallazgo, la Fundación Ortopédica de Animales (OFA) identificó a los perros molosoides como el tipo de canes con más alta frecuencia de presentación. Algunos ejemplos de esta relación son el Dogue de Bordeaux (57,4\%), Perro de San Bernardo (49,1\%) y el Mastín Napolitano (51,3\%) (Comhaire y Snaps, 2008; Orthopedic Foundation for Animals [OFA], 2017).

Estas condiciones ocasionarán una subluxación de la cabeza femoral durante la carga de peso. A lo largo del tiempo esto conducirá a un desgaste anormal de las superficies articulares y al desarrollo de la enfermedad articular degenerativa así como la exostosis (crecimiento anormal del hueso) y la erosión del cartílago. (Ginja et al., 2008). La DC con el tiempo terminará resultando en una enfermedad secundaria de la articulación (artritis, artrosis) ocasionando signos clínicos como dolor y cojera. Los signos clínicos pueden aparecer desde los cinco meses de edad afectando la movilidad y el bienestar del animal por el resto de su vida. Aunque la mayoría de los animales afectados no muestran signos o lo hacen en grados muy pequeños por lo que muchas veces hace difícil reconocer la enfermedad en sus inicios (Woolliams et al., 2011).

La enfermedad se puede evaluar y diagnosticar física-clínicamente, sin embargo, la evaluación radiológica es la base para la valoración definitiva de enfermedad en caninos (Brass y Paatsama, 1983). El examen radiográfico ha sido usado por más de 40 años para ayudar no solo en el diagnóstico de la enfermedad sino también a los programas de cría seleccionando a los reproductores (Guo et al., 2011; Woolliams et al., 2011). De esta forma, para disminuir la prevalencia de la enfermedad, las principales organizaciones de criadores de canes: Fédération Cynologique Internationale, the Orthopedic Foundation for Animals y la British Veterinary Association/Kennel Club, usan el método radiográfico de la incidencia ventral con miembros extendidos para clasificar posibles perros aptos para la reproducción (Broeckx et al., 2014). La severidad será juzgada radiográficamente en base al grado de subluxación de la cabeza femoral, además de la profundidad y congruencia del acetábulo y la presencia de malformaciones articulares secundarias (Flückiger, 2007).

En el presente trabajo se determinó radiográficamente la frecuencia de presentación de DC en una raza susceptible como el Mastín Napolitano. Así mismo, se evaluó su grado de asociación con las variables sexo, grupo etáreo y signos clínicos.

\section{MATERIAL Y MÉTODOS}

\section{Lugar de estudio y pacientes}

El trabajo se realizó en una Clínica Veterinaria de Lima Metropolitana con un equipo de rayos X General Electric modelo móvil 225, de columna móvil con capacidad máxima de 125 Kvp y 200 miliamperios. La muestra de pacientes correspondió a los caninos de la raza Mastín Napolitano (machos y hembras) que llegaron a la Clínica ya sean por consultas, ecografías, servicios de rayos $\mathrm{X}$, etc. Los pacientes fueron mayores de 6 meses de edad y su participación fue debidamente autorizada por sus propietarios. Previo a el examen radiográfico, se administró vía endovenosa clorhidrato de Ketamina (10 mg/kg p. v.) y diazepam $(0,05 \mathrm{mg} / \mathrm{kg}$ 
p.v.), con fines de facilitar el posicionamiento de los pacientes y mejorar la calidad de la imagen.

\section{Evaluación físico-clínica}

Se confeccionó una historia clínica y se realizó a los propietarios de los pacientes una anamnesis completa, seguidamente se realizó el examen físico en el cual se observó al paciente in situ (figura 1), al caminar y al trote. Finalmente una vez sedados, se procedió a realizar un completo examen ortopédico y el test de Ortolani (figura 2), el cual se consideró positivo cuando hubo reintroducción de la cabeza femoral en el acetábulo luego de la abducción del miembro evaluado (Fossum, 2009).

\section{Evaluación radiográfica}

Los pacientes se ubicaron en posición decúbito dorsal con la región torácica ubicada en un posicionador para restringir la rotación lateral. La incidencia radiografía necesaria fue la ventro dorsal, una vez sedado el paciente, los miembros anteriores se proyectaron hacia craneal ubicando la cabeza entre ellos. Los miembros posteriores se traccionaron en dirección caudal, llevando el eje medio femoral paralelo al chasis radiográfico, ubicando ambos miembros paralelos entre si y girando las rodillas hacia adentro. Se distinguió mediante marcadores de plomo el lado derecho de la placa radiográfica. La técnica radiológica, miliamperios y kilovoltio potencia fueron reguladas según el protocolo de exposición en pequeños animales planteados por Thrall (2009).

El diagnóstico radiográfico se realizó según el procedimiento radiográfico para la evaluación de la DC de la Federación Cinológica Internacional (FCI), sobre la base de los parámetros anátomo radiográficos y la determinación del ángulo de Norberg (AN). Este se calculó a partir de la unión de los centros de las cabezas femorales por intermedio de una línea, y la proyección de una segunda línea trazada a partir de cada uno de estos centros hasta una tangente del respectivo borde acetabular cráneo-lateral (figura 3). El ángulo interno entre las líneas es medido, el menor ángulo compatible con la normalidad es de $105^{\circ}$ (Comhaire y Schoonjans, 2011).

\section{Datos y Análisis Estadístico}

Se identificó la frecuencia de presentación de DC, su distribución por sexo, estrato etáreo y la distribución de los casos según modo de afección (unilateral o bilateral). Se determinó la asociación estadística entre la presentación de la enfermedad y el sexo evaluado mediante la prueba de Chi cuadrado. Se presentó la frecuencia de presentación de DC, por articulaciones coxofemorales evaluadas, según los grados de
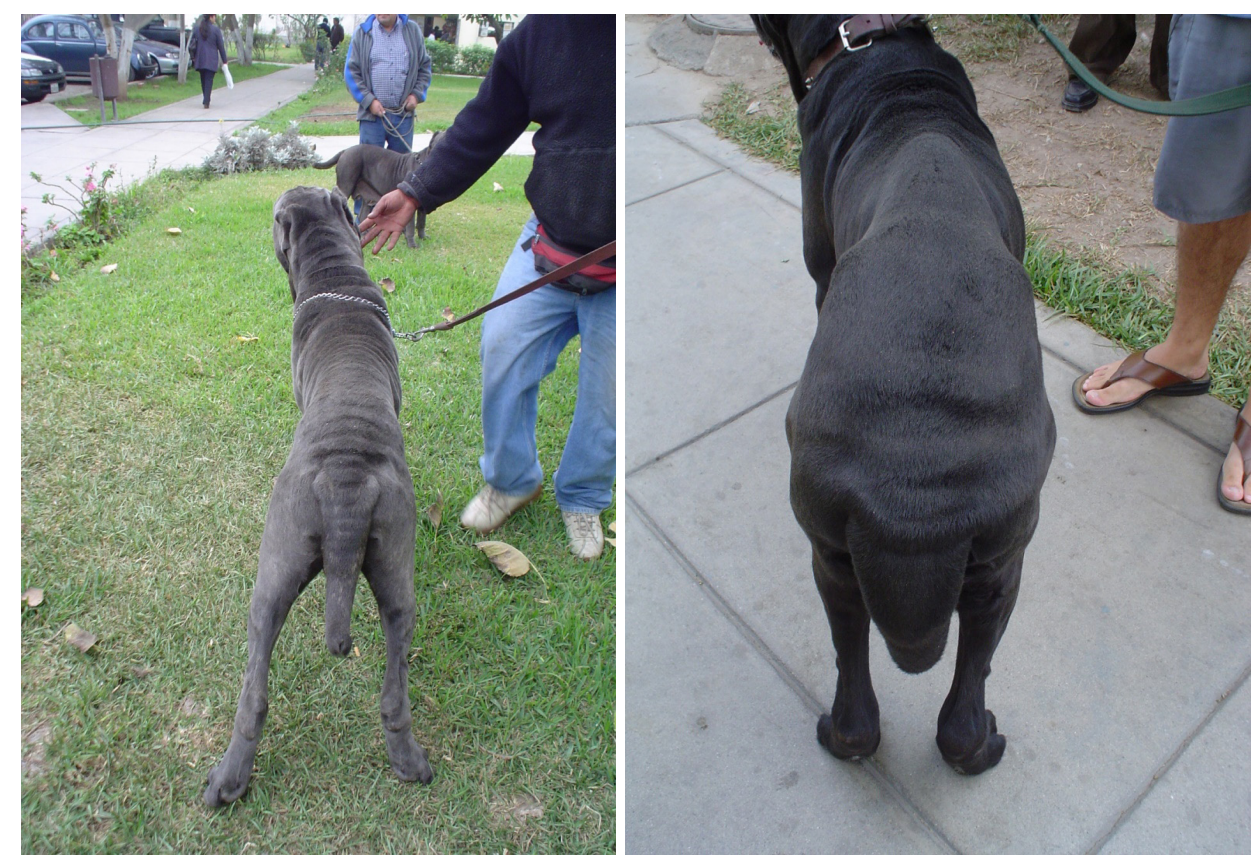

Figura 1. Examen físico (inspección) en un Mastín Napolitano con severa DC (derecha): presenta atrofia de la musculatura de la cadera y miembros posteriores además de base de sustentación estrecha. Figura izquierda: Buena conformación y masa muscular homogénea de la cadera y tren posterior en un paciente libre de DC. 

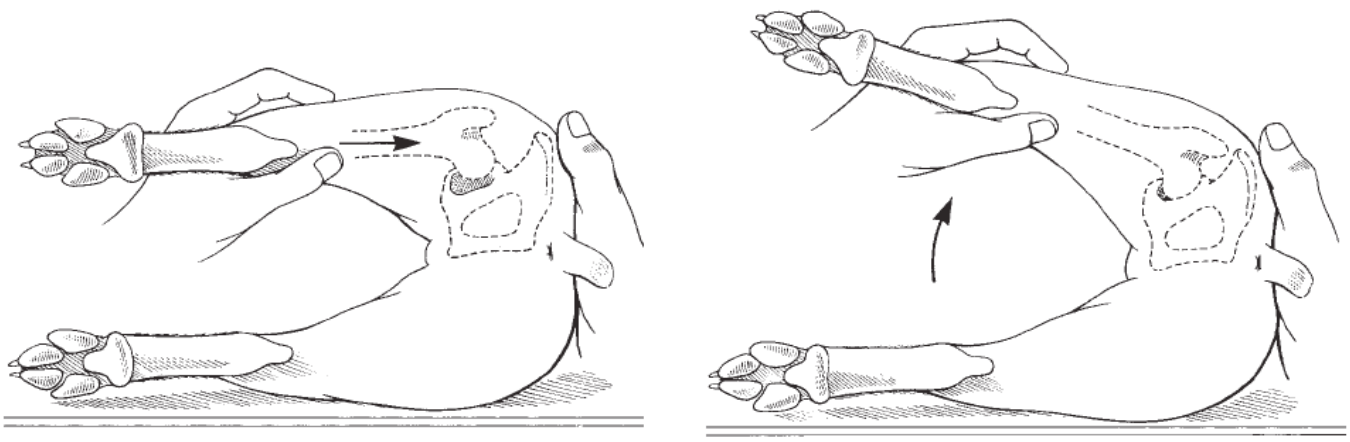

Figura 2. Test de ortolani con el paciente en decúbito lateral (izquierda): se coloca una mano sobre la espalda del paciente, y se sujeta la rodilla con la mano libre. En un solo movimiento se debe producir subluxación empujando la rodilla hacia la pelvis. (Derecha): Manteniendo la presión se produce la aducción del miembro. Si se escucha un crepitar al reintroducir la cabeza en el acetábulo se considera positivo (Fossum, 2009).

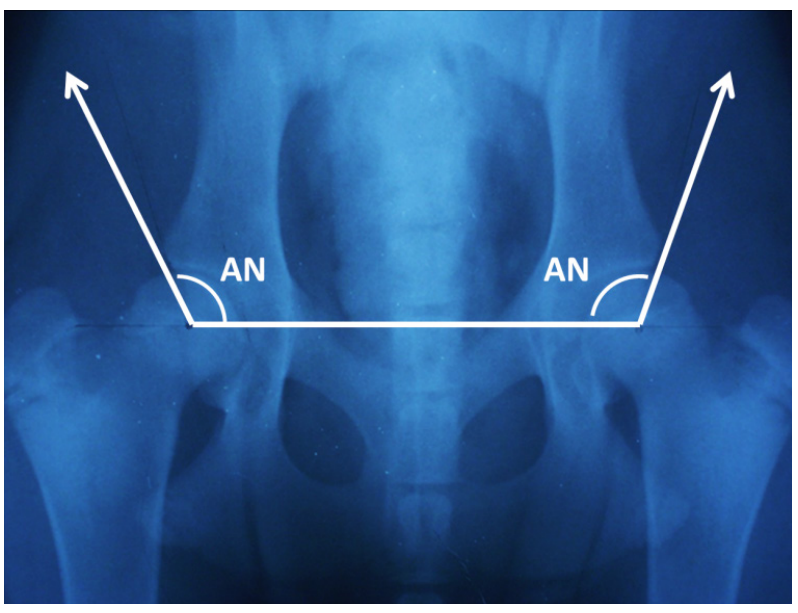

Figura 3. Método radiográfico (incidencia ventro dorsal con los miembros extendidos), según la FCI para la evaluación de DC en canes, mediante el ángulo de Norberg (AN).

afección de acuerdo a las normas internacionales de la FCI. Se determinó la asociación entre los pacientes positivos a displasia por el método radiológico y los signos clínicos, mediante la prueba no paramétrica de correlación de Spearman.

\section{RESULTADOS}

Se obtuvo una frecuencia de presentación de DC de $65 \%$ diagnosticándose 39 canes positivos sobre un total de 60 canes. El 70\% de los machos y el $60 \%$ de las hembras presentaron algún grado de DC. No se obtuvo diferencias estadísticas significativas para la variable sexo, evaluados por la prueba Chi cuadrado. La mayor proporción de animales positivos a DC ocurrió en animales entre 6 y 18 meses de edad. No hubo diferencias estadísticas entre grupos por edad (tabla 1).
En el presente trabajo, se encontró una mayor proporción estadísticamente significativa de animales afectados en forma bilateral 69,2\% (27/39) (tabla 2).

El $53,8 \%$ de los casos diagnosticados positivos radiográficamente (21/39), presentaron signos clínicos luego de realizarse el examen ortopédico como: base de sustentación estrecha, crepitación articular y dolor a la extensión de la cadera. Además, fueron positivos a la prueba de Ortolani. Se observó asociación estadística significativa entre el modo de presentación bilateral y la presencia de signos clínicos (tabla 3).

Tabla 1. Distribución de los resultados de displasia de cadera según la edad, en caninos de la raza Mastín Napolitano.

\begin{tabular}{lccc}
\hline & & \multicolumn{2}{c}{ Positivo } \\
Edad meses & Animales & $\mathrm{n}$ & $(\%)$ \\
\hline De $6 \mathrm{~m} \mathrm{a}<18 \mathrm{~m}$ & 34 & 23 & 67,6 \\
De $18 \mathrm{~m} \mathrm{a}<60 \mathrm{~m}$ & 22 & 16 & 72,7 \\
\cline { 3 - 4 }$\geq$ a $60 \mathrm{~m}$ a más & 4 & 0 & 0 \\
Total & 60 & 39 & 65 \\
\hline Nota: No existe diferencia estadística significativa & &
\end{tabular}

Tabla 2. Distribución de los casos de displasia de cadera según modo de afección, en caninos de raza Mastín Napolitano

\begin{tabular}{lll}
\hline Variable & \multicolumn{2}{l}{ Animales } \\
\hline \multirow{2}{*}{$\begin{array}{ll}\text { Articulación afectada } \\
\text { Ninguna }\end{array}$} & Evaluados (n) & Afectados (\%) \\
\cline { 2 - 3 } Unilateral & 21 & $0^{\mathrm{a}}$ \\
Bilateral & 12 & $30,8^{\mathrm{a}}$ \\
Total & 27 & $69,2^{\mathrm{b}}$ \\
& 60 & 100
\end{tabular}

Nota: ${ }^{\mathrm{a}, \mathrm{b}}$ Letras diferentes indican que las proporciones en cada fila son estadísticamente diferentes $(p<0.05)$ 
Elmayorporcentajedearticulacionescoxofemorales afectadas con DC fueron diagnosticados con grado D o displasia media con 18,3\% (22/120), en tanto que la menor frecuencia de presentación correspondió al grado E o displasia grave 10\% (12/120) (tabla 4, figura 4 y figura 5).

\section{DISCUSIÓN}

En el presente trabajo, se encontró en una alta frecuencia la presentación de DC (65\%), en comparación con las evaluaciones realizadas por la OFA, los cuales determinaron una presentación de DC en el 51,3\% de los casos (OFA, 2017). Esta frecuencia nos da una referencia importante y actualizada del comportamiento y características de presentación de la enfermedad en la ciudad de Lima Perú y en una raza altamente susceptible como el Mastín Napolitano (Salinas, 2006).

Cabe destacar que si bien la relevancia del método del AN ha sido cuestionada por Smith et al., (1993). En publicaciones posteriores diversos autores respaldan la capacidad de este método radiográfico para predecir el riesgo de sufrir enfermedad degenerativa articular

Tabla 3. Frecuencia de presentación de signos clínicos* en caninos positivos radiológicamente a displasia de cadera.

\begin{tabular}{lccc}
\hline Modo de afección & Signos clínicos* & Evaluados (n) & Afectados (\%) \\
\hline Unilateral & 4 & 12 & $33,3^{\mathrm{a}}$ \\
Bilateral & 17 & 27 & $63{ }^{\mathrm{b}}$ \\
Total & 21 & 39 & 53,8 \\
\hline a,b Letras diferentes indican que las proporciones en cada fila son estadísticamente \\
diferentes ( $<<0.05) .{ }^{*}$ Luego de la evaluación ortopédica y prueba de Ortolani.
\end{tabular}

Tabla 4. Clasificación y frecuencia de presentación por articulaciones coxofemorales evaluadas, según grados de afección (Federación Cinológica Internacional).

\begin{tabular}{lcc}
\hline Grados de displasia & Articulaciones $\mathrm{n}$ & Afectados (\%) \\
\hline (A) Normal & 53 & 44,2 \\
(B)Transición & 15 & 12,5 \\
(C) Leve & 18 & 15 \\
(D) Media & 22 & 18.3 \\
(E) Grave & 12 & 10 \\
Total & 120 & 100 \\
\hline
\end{tabular}

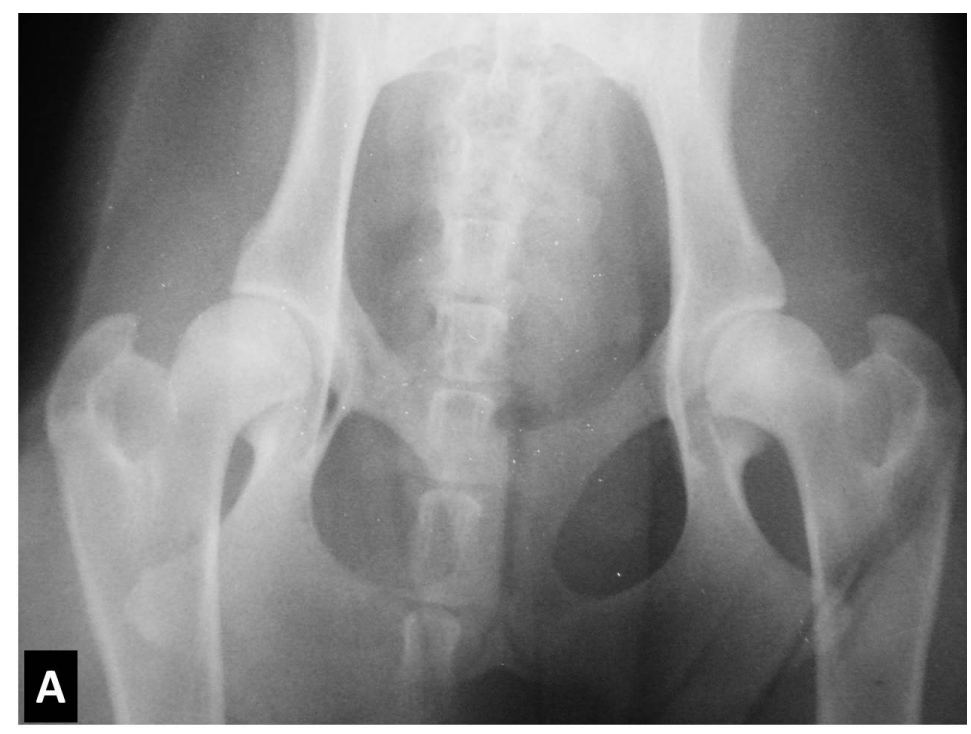

Figura 4. Libre de displasia de cadera. 


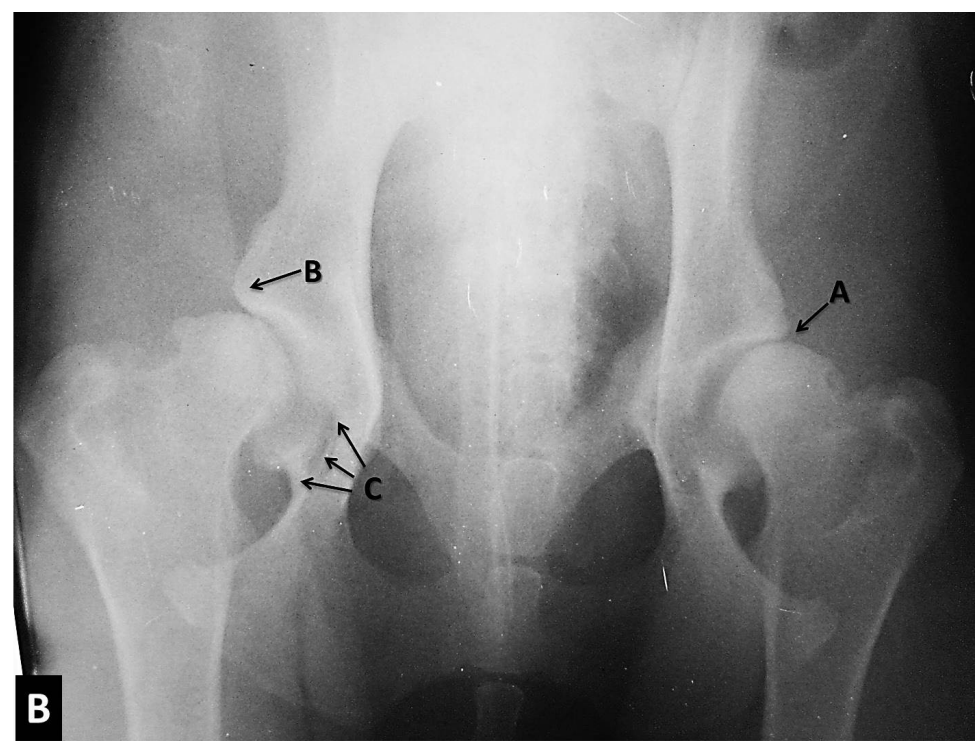

Figura 5. Grave DC bilateral (Grado E). a. Subluxación de la cabeza femoral; b. Aplanamiento de la ceja acetabular craneal; c. Marcada laxitud articular con degeneración y pérdida de contornos articulares.

(Banfield et al., 1996; Malm et al., 2010) y contribuir a la reducción de la prevalencia de la enfermedad en perros de pedrigree (Comhaire y Schoonjans, 2011).

El sexo de los animales no estuvo asociado a la presentación de la enfermedad, en este sentido Smith (1997), tampoco encontró diferencia significativa entre sexos. En el Perú, Tong (2000) en Labradores Retriever, y Dávila, Fernández, Chavera y Díaz (2002), en Rottweilers, tampoco encontraron diferencias significativas. En contraste, Thibaut, Gotschlich, Mansilla, Campillo y Vargas (2005) en un trabajo realizado en ovejeros alemanes, los perros machos presentaron diferencias estadísticamente significativas de DC respecto de las hembras. Estos resultados obtenidos pueden deberse a la mayor edad, peso y alzada promedio que tuvieron los machos respecto a las hembras. Según Lust, Rendano y Summers (1985), estos factores pueden ser determinantes en la presentación de DC.

La distribución de los resultados según distribución por edades, indicó que no existe asociación entre presentación de la enfermedad y la edad. Estos resultados corroboran la información presentada por Smith et al., (2001). Distinguiendo que la enfermedad se puede presentar en animales tan jóvenes (menos de 6 meses) y mayores (5 años a más). La edad no sería un factor que desencadene la enfermedad sin embargo debe considerarse para el examen radiográfico, ya que si se realiza antes de los dos años, la efectividad del diagnóstico puede ser menor. En un trabajo utilizando la evaluación fenotípica de las radiografías de caderas por la OFA, Corley, Keller, Lattimer y Ellersieck (1997) reportaron una efectividad diagnostica del $69,9 \%$ a los 12 meses, un $82,7 \%$ a los 18 meses y un $95,4 \%$ a los 2 años de edad. En general no se declara libre de DC a perros menores de dos años (Thrall, 2009). Si bien es cierto la DC se considera una enfermedad degenerativa asociado al desarrollo, no siempre se manifiestan signos morbosos a temprana edad.

La manifestación de la enfermedad dependerá principalmente de los factores ambientales, nutricionales así como del grado de actividad física de los pacientes. Un estudio realizado en perros de raza Labrador Retriever alimentados con un 25\% menos de ración que los controles, presentaron una reducción en la frecuencia de presentación de la enfermedad. Esto se explicaría por la disminución en el ritmo de crecimiento, ya que en animales alimentados con dietas restringidas, los núcleos de osificación acetabular se osificarán antes de los 7 meses. De manera contraria, con un ritmo de crecimiento mayor, estos núcleos de osificación pélvica se cerraran a los 6 meses, esto podría contribuir al desarrollo de DC (McLaughlin y Tomlinson, 1996).

La enfermedad se mostró mayoritariamente bilateral manifestando los signos radiográficos como inestabilidad articular en casos leves y marcados cambios degenerativos como subluxación, remodelación articular y otros signos de enfermedad 
degenerativa articular. Los casos unilaterales correspondieron generalmente con presentaciones iniciales de la enfermedad y en animales jóvenes con signos de laxitud articular la cual no necesariamente mostraba marcados signos osteoartríticos, esta característica está descrita por Kyriazis (2016).

Se observó asociación significativa entre la presentación de signos clínicos y su relación con caninos positivos radiológicamente a DC, esta relación se presentó principalmente en los pacientes con displasia bilateral. Históricamente, muchos perros con DC no muestran signos clínicos y su presentación puede ser muy relativa ya que es importante que el Médico Veterinario tenga la experiencia necesaria para desarrollar adecuadamente el examen ortopédico. En el presente estudio, este hallazgo tendría relación con los cambios articulares producidos por la enfermedad avanzada, como subluxación y osteoartritis. Estos eventos que al desarrollar cronicidad producen incapacidad motora y funcional debido al dolor.

En el presente estudio, el grado de afección D (Media), fue el de mayor presentación. Estos resultados son considerados elevados en comparación con los datos internacionales de la OFA (2017). En la evaluación radiológica de los casos positivos, se describió animales con signos comunes como: Subluxación de cabeza femoral, remodelación articular con grados de degeneración y aplanamiento de la ceja acetabular craneodorsal frecuentemente asociado a osteofitos peri articulares (Tobias y Johnston, 2013). Por otra parte, la laxitud articular, fue el hallazgo más común en los animales positivos. Este signo se presentó aun en animales jóvenes de 6 meses, en los cuales se asoció con subluxación de cabeza femoral en los grados de DC avanzada.

Habría que añadir que los animales considerados en el estudio no fueron muestreados exclusivamente por presentar $\mathrm{DCl}$, lo que contribuye a demostrar en nuestro medio una probable presentación subclínica en las poblaciones de perros de la raza Mastín Napolitano así como en razas de características similares. Es importante destacar que un gran número de los ejemplares que ingresaron al estudio tenían registro de raza vigente expedido, y con un estado de salud aceptable.

\section{CONCLUSIONES}

Se determinó un $65 \%$ de presentación de displasia de cadera en canes Mastín Napolitano, considerada elevada en comparación con los datos registrados internacionalmente por la Fundación Ortopédica de Animales.

La enfermedad no presentó asociación con el sexo y la edad de los pacientes.

La presentación de la enfermedad fue mayoritariamente bilateral $(69, \%)$ y altamente asociada a signos clínicos $(63 \%)$.

El mayor grado de afección según parámetros de la FCI correspondió al grado de afección D (Media) entre los animales positivos radiológicamente.

\section{Correspondencia}

Eben Salinas

Correo electrónico: esalinasc@cientifica.edu.pe

\section{REFERENCIAS BIBLIOGRAFICAS}

1. Banfield, C. M., Bartels, J. E., Hudson, J. A., Wright, J. C., Hathcock, J. T. \& Montgomery, R. D. (1996). A retrospective study of canine hip dysplasia in 116 military working dogs: Part I: angle measurements and Orthopedic Foundation for Animals (OFA) grading. Journal American Animal Hospital Association, 32, 413-422.

2. Brass, W. \& Paatsama, S. (1983). Hip Dysplasia International certificate and evaluation of radiographs. Helsinki: FCI. p. 1- 25.

3. Broeckx, B. J. G., Verhoeven, G., Coopman, F., Van Haeringen, W., Bosmans, T., Gielen, I., ... \& Verbeke, V. (2014). The effects of positioning, reason for screening and the referring veterinarian on prevalence estimates of canine hip dysplasia. The Veterinary Journal, 201(3), 378-384.

4. Cardinet G. H., Kass, P. H., Wallace, L. J., \& Guffy, M. M. (1997). Association between pelvic muscle mass and canine hip dysplasia. Journal of the American Veterinary Medical Association, 210(10), 1466-1473.

5. Comhaire, F. H., \& Schoonjans, F. A. (2011). Canine hip dyslasia: the significance of the Norberg angle for healthy breeding. Journal of small animal practice, 52(10), 536-542.

6. Comhaire, F. H., \& Snaps, F. (2008). Comparison of two canine registry databases on the prevalence of hip dysplasia by breed and the relationship of dysplasia with body weight and height. American journal of veterinary research, 69(3), 330-333.

7. Corley, E. A., Keller, G. G., Lattimer, J. C., \& Ellersieck, M. R. (1997). Reliability of early radiographic evaluations for canine hip dysplasia 
obtained from the standard ventrodorsal radiographic projection. Journal of the American Veterinary Medical Association, 211(9), 1142-1146.

8. Dávila, F., Fernández, A., Chavera, C., \& Díaz, C. (2002). Frecuencia de displasia de cadera en caninos Rottweiler mayores de un año de edad. Revista de Investigaciones Veterinarias del Perú, 13(1), 32-37.

9. Flückiger, M. (2007). Scoring radiographs for canine hip dysplasia-The big three organizations in the world. European Journal of Companion Animal Practice, 17, 135-140.

10. Fossum TW. (2009). Cirugía en pequeños animales. Barcelona: Elsiever. pp.1233- 1235.

11. Ginja, M. M. D., Gonzalo-Orden, J. M., Melo-Pinto, P., Bulas-Cruz, J., Orden, M. A., San Roman, Ferreira, A. J. A. (2008). Early hip laxity examination in predicting moderate and severe hip dysplasia in Estrela mountain dog. Journal of Small Animal Practice, 49(12), 641-646.

12. Ginja, M. M. D., Silvestre, A. M., Gonzalo-Orden, J. M., \& Ferreira, A. J. A. (2010). Diagnosis, genetic control and preventive management of canine hip dysplasia: a review. The Veterinary Journal, 184(3), 269-276.

13. Guo, G., Zhou, Z., Wang, Y., Zhao, K., Zhu, L., Lust, G., ... \& Harris, S. (2011). Canine hip dysplasia is predictable by genotyping. Osteoarthritis and cartilage, 19(4), 420-429.

14. Kyriazis, A. (2016). Canine hip dysplasia. Part I: Aetiopathogenesis \& diagnostic approach. Hellenic $J$ Companion Anim Med, 5(1), 36-47.

15. Lust, G., Rendano, V. T., \& Summers, B. A. (1985). Canine hip dysplasia: concepts and diagnosis. Journal of the American Veterinary Medical Association, 187(6), 638-640.

16. Malm, S., Fikse, F., Egenvall, A., Bonnett, B. N., Gunnarsson, L., Hedhammar, A. \& Strandberg, E. (2010). Association between radiographic assessment of hip status and subsequent incidence of veterinary care and mortality related to hip dysplasia in insured Swedish dogs. Preventive Veterinary Medicine, 93, 222-232

17. Mclaughlin, R., \& Tomlinson, J. (1996). Radiographic diagnosis of canine hip dysplasia. Veterinary Medicine, 91(1), 36.
18. Orthopedic Foundation for Animals (2017). Statistics by Disease \& Breed. Columbia : Orthopedic Foundation for Animals. Recuperado de https://www. ofa.org/diseases/breed-statistics\#detail

19. Salinas, E. (2006). Frecuencia de presentación de displasia coxofemoral en canes de la raza Mástil [i.e. Mastín] napolitano. Tesis de Pregrado. Universidad Nacional Mayor de San Marcos. Recuperado de http://alicia.concytec.gob.pe/vufind/ Record/UNMS_798457177ee2e858b950d4376e781 6aa/Details?cv $=1$

20. Smith, G. K. (1997). Advances in diagnosing canine hip dysplasia. Journal of the American Veterinary Medical Association, 210(10), 1451-1457.

21. Smith, G. K., Mayhew, P. D., Kapatkin, A. S., McKelvie, P. J., Shofer, F. S., \& Gregor, T. P. (2001). Evaluation of risk factors for degenerative joint disease associated with hip dysplasia in German Shepherd Dogs, Golden Retrievers, Labrador Retrievers, and Rottweilers. Journal of the American Veterinary Medical Association, 219(12), 1719-1724.

22. Thibaut, J., Gotschlich, E., Mansilla, M., Campillo, C., \& Vargas, L. (2005). Clinical-radiographic diagnosis of hip dysplasia in German shepherd dogs from Valdivia City, Chile. Revista Cientifica-Facultad de Ciencias Veterinarias, 15(2), 125-133.

23. Thrall, D.E. (2009). Tratado de diagnóstico radiológico veterinario. Buenos Aires: Intermedica. pp 584-606.

24. Tobias, K. M., \& Johnston, S. A. (2013). Veterinary surgery: small animal: 2-Volume Set. Elsevier Health Sciences.

25. Tong, J.C. (2000). Estudio radiológico sobre la frecuencia de presentación de displasia de cadera en caninos de la raza Labrador Retriever. Tesis para optar al grado de Médico Veterinario. Universidad Nacional Mayor de San Marcos. Lima, Perú. 4-14 pp.

26. Witsberger T.,Villamil A., Schultz L., Hahn A., \& Cook J. (2008). Prevalence of and risk factors for hip dysplasia and cranial cruciate ligament deficiency in dogs. Journal of the American Veterinary Medical Association, 232 (12), 1818-1824.

27. Woolliams, J. A., Lewis, T. W., \& Blott, S. C. (2011). Canine hip and elbow dysplasia in UK Labrador retrievers. The Veterinary Journal, 189(2), 169-176. 